\title{
On the Geographic Asymmetry of Typhoon Translation Speed across the Mountainous Island of Taiwan
}

\author{
Li-HuAn Hsu AND Hung-CHI KuO \\ Department of Atmospheric Sciences, National Taiwan University, and Taiwan Typhoon and Flood Research Institute, \\ Taipei, Taiwan \\ ROBERT G. FOVELL \\ Department of Atmospheric and Oceanic Sciences, University of California, Los Angeles, Los Angeles, California
}

(Manuscript received 14 June 2012, in final form 7 August 2012)

\begin{abstract}
This paper examines the effect of topographically phase-locked convection on the motion of typhoons across the island of Taiwan. Data for 84 typhoons that reached Taiwan's eastern coast from 1960 to 2010 are analyzed, with motions compared to the long-term average overland translation speed. For 61 continuoustrack typhoons among all cases, $77 \%$ of the slow-moving tropical cyclones (TCs) made landfall on the northern end of Taiwan's eastern coast, while $60 \%$ of the fast storms had southeastern coastal landfalls.

This geographic asymmetry with respect to typhoon translation speeds widened after landfall, as the slow movers typically decelerated during the overland period, whereas the faster TCs sped up. In particular, the average overland duration was $16 \mathrm{~h}$ for the slow class, compared to only $3 \mathrm{~h}$ for the fast-moving typhoons. The combination of slower translation with longer duration for the northern class of TCs led to large rainfall on the southwestern slope of the island's Central Mountain Range.

Weather Research and Forecasting model experiments are used to study the effect of convection on storm motion over a mountainous island resembling Taiwan. The authors find that the topographically phase-locked convection acts to slow down (speed up) the northern (southern) landfalling typhoons. The model results also suggest that a positive feedback mechanism exists for the slow storms, in which the convective heating pattern forced by topography acts to reduce the TC motion, leading to even more prolonged precipitation and heating, yielding further speed reductions.
\end{abstract}

\section{Introduction}

Taiwan is a mountainous island oriented nearly north-south and is $150 \mathrm{~km}$ in width and $400 \mathrm{~km}$ in length, with the peak altitude of its Central Mountain Range (CMR) exceeding $3000 \mathrm{~m}$. The annual precipitation of Taiwan is approximately $2500 \mathrm{~mm}$, with most of the heavy rainfall resulting from landfalling typhoons, when and where the air is forced to rise over the range (Chang 1982). The typhoon rainfall pattern is phase locked with the CMR, with its southwestern slope receiving especially large amounts (Chang et al. 1993). It is reasonable that the more slowly storms move and/or the

Corresponding author address: Robert G. Fovell, Department of Atmospheric and Oceanic Sciences, University of California, Los Angeles, Los Angeles, CA 90095-1565.

E-mail: rfovell@ucla.edu longer time they spend over land, the more precipitation can fall on the island-a striking example of this being Typhoon Morakot (2009). While not a notable storm with respect to maximum wind speeds, Morakot's slow translation speed and ample moisture supplied by the southwest monsoon are two key reasons why that typhoon produced the largest recorded rainfall in Taiwan in the past $51 \mathrm{yr}$ (Chien and Kuo 2011).

The expected relationship between rainfall and typhoon motion is confirmed in Fig. 1, which presents the total rainfall amount recorded at 21 Central Weather Bureau (CWB) surface stations for a set of 61 continuoustrack (defined presently) typhoons that made landfall on Taiwan's eastern coast in the 1960-2010 period. Total typhoon rainfall is plotted against the reciprocal of land translation speed (Fig. 1a) and the overland duration time (Fig. 1b). There are 40 northern landfall typhoons (NLT; dots) and 21 southern landfall typhoons 
(a)

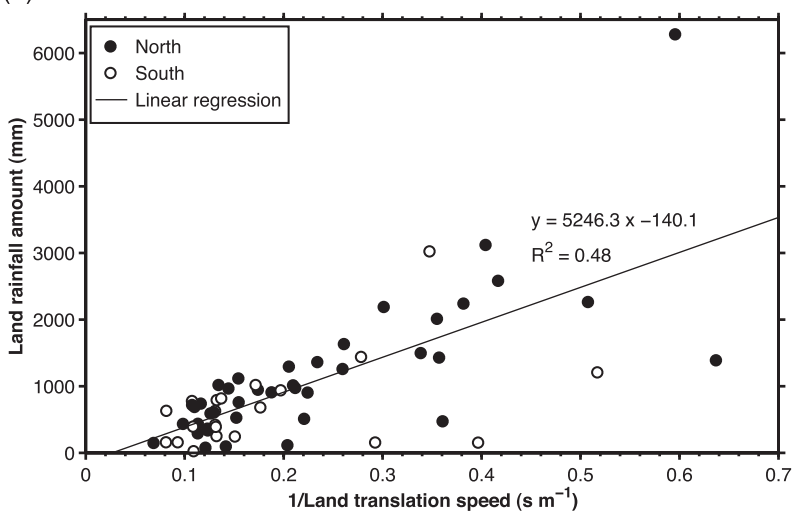

(b)

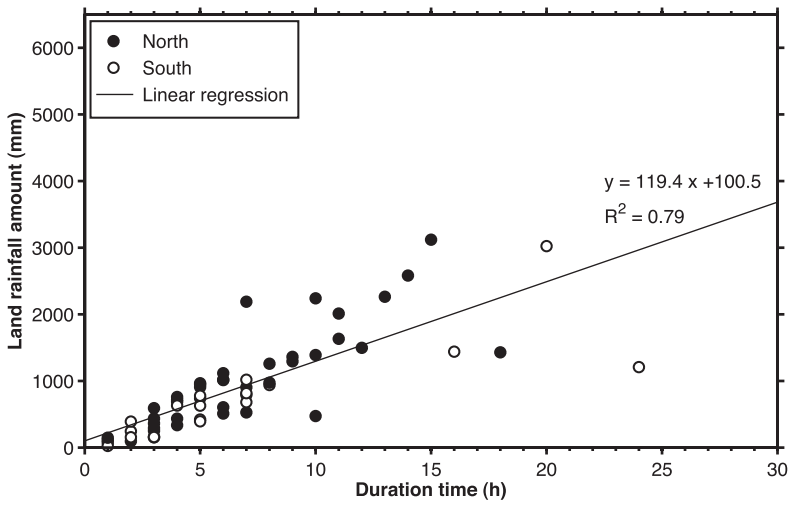

FIG. 1. Observed rainfall amount vs (a) the reciprocal of overland translation speed and (b) overland duration time, for 61 continuous-track typhoons that made landfall on the Taiwan eastern coast between 1960 and 2010. Northern and southern groups are represented as dots and circles, respectively. Solid lines are least squares fits using all cases.

(SLT; circles), based on where their centers reached Taiwan's eastern coast relative to $23.5^{\circ} \mathrm{N}$ (Table 1 ). While the overall relationship between rainfall and the two independent variables is similar for the NLT and SLT classes (not shown), it is also clear that the heaviest rainfall events are more likely to have a landfall on the northern segment of the eastern coast, and those cases are more likely to be slower moving and have longer overland durations (Table 1). Thus, there appears to be a marked geographic asymmetry with respect to both motion and rainfall production.

Numerous studies have examined the impact of the CMR on landfalling tropical cyclones (TCs). For example, Brand and Blelloch (1974) found a significant $40 \%$ reduction in typhoon intensity after landfall, while Ishijima and Estoque (1987) noted the formation of secondary lows west of the CMR for some westbound storms. The numerical results in Chang (1982) suggested that secondary vortex centers formed on the lee side as the typhoon is blocked upstream by the CMR. Wang (1980) and Shieh et al. (1998) studied the Taiwan orography effect on the movement with more than 200 landfalling typhoons between 1897 and 1996. They further suggested that the leeside secondary low could sometimes fully replace the original typhoon center, resulting in "discontinuous tracks."

When translation speed is defined based on the surface vortex signature, a discontinuous motion may appear as a very fast translation speed. Yeh and Elsberry (1993a,b) studied upstream deflections as well as continuous and discontinuous tracks for westward-moving tropical cyclones passing Taiwan. They found that, in agreement with the observations (e.g., Shieh et al. 1998), typhoons tend to track continuously across the northern end of the island but that discontinuous tracks dominate for weaker typhoons that made landfall at central or southern Taiwan. More intense and rapidly moving storms may cross directly over the barrier. It is likely that the greater frequency of discontinuous tracks in the south contributes to the fast speed of the SLT class. However, we will show in section 2 that the SLT are still characterized by rapid motions even when the discontinuoustrack typhoons are excluded.

Tracks with cyclonic deflections sometimes appear before landfall, accompanied with along-track acceleration

TABLE 1. The mean and standard deviation of duration, translation speed, distance, and rainfall amount during the overland period. In total, 61 continuous-track storms between 1960 and 2010 are categorized as NLT or SLT based on landfall position relative to $23.5^{\circ} \mathrm{N}$. Fast, medium, and slow groups are classified relative to the mean and standard deviation of the overland translation speed; see text for details.

\begin{tabular}{|c|c|c|c|c|c|c|c|c|}
\hline \multirow[b]{2}{*}{ Category (numbers) } & \multicolumn{2}{|c|}{$\begin{array}{c}\text { Overland } \\
\text { duration }(\mathrm{h})\end{array}$} & \multicolumn{2}{|c|}{$\begin{array}{l}\text { Overland translation } \\
\text { speed }\left(\mathrm{m} \mathrm{s}^{-1}\right)\end{array}$} & \multicolumn{2}{|c|}{$\begin{array}{c}\text { Overland } \\
\text { distance }(\mathrm{km})\end{array}$} & \multicolumn{2}{|c|}{$\begin{array}{c}\text { Rainfall amount } \\
\text { while overland (mm) }\end{array}$} \\
\hline & Mean & Std dev & Mean & Std dev & Mean & Std dev & Mean & Std dev \\
\hline NLT (40) & 7.9 & 8.1 & 5.7 & 2.9 & 116.7 & 48.5 & 1157.4 & 1101.3 \\
\hline SLT (21) & 6.6 & 6.1 & 7.0 & 3.0 & 128.4 & 59.8 & 680.9 & 659.9 \\
\hline Fast (10) & 3.4 & 1.4 & 10.6 & 1.9 & 125.7 & 47.9 & 413.1 & 279.0 \\
\hline Slow (13) & 16.2 & 11.7 & 2.4 & 0.5 & 132.9 & 71.7 & 2128.3 & 1533.3 \\
\hline Medium north (26) & 5.4 & 2.5 & 6.2 & 1.8 & 110.7 & 39.5 & 808.5 & 498.7 \\
\hline Medium south (12) & 5.9 & 3.9 & 6.3 & 1.6 & 125.1 & 59.5 & 647.8 & 378.7 \\
\hline DIS (23) & 5.7 & 5.4 & 18.7 & 16.5 & 192.9 & 68.0 & 560.7 & 557.2 \\
\hline
\end{tabular}


(Bender et al. 1987). Yeh and Elsberry (1993a) showed that the Taiwan terrain may enhance the southward flow on the western side of the typhoon circulation via the channel effect and thus further influence the typhoon track, a result also reported by Jian and Wu (2008). Kuo et al. (2001) suggested that the topographic vortexstretching effect, which is due to the storm circulation crossing the CMR, may also induce the NLT to shift southward before landfall. The topographic $\beta$ effect on tropical cyclone motion has also been shown to be important in other areas with a large-scale topography, such as the Sierra Madre (Zehnder 1993). In summary, the southward deflection track or looping before landfall for NLT, in general, may lead to a larger rainfall event in Taiwan before the typhoon landfall at Taiwan (Su et al. 2012).

Herein, we pursue a different and/or complementary approach and hypothesize that convective activity forced by the CMR has a material impact on the track of a typhoon as it approaches and passes the island, which we will demonstrate using a potential vorticity $(\mathrm{PV})$ tendency analysis following Wu and Wang (2000, hereafter WW00). Typhoons represent local maxima of PV and tend to move toward the maximum of the azimuthal wavenumber-1 part of PV tendency (WW00). The net tendency reflects contributions from horizontal and vertical PV advection as well as friction and gradients of diabatic heating. Therefore, as shown by Chan et al. (2002), the presence of asymmetric convection in a typhoon may have an influence on the track, if it has a significant wavenumber-1 component. While external forcings such as vertical wind shear can influence convective asymmetries (Corbosiero and Molinari 2003), our interest here is in asymmetries associated with the atmospheric response to the CMR.

We further hypothesize that as a typhoon approaches and passes Taiwan, the impact of asymmetric convection on its motion changes, precisely because convection over the CMR is phase locked with the topography. As a TC approaches the island from the east or southeast, its circulation combines with the orographic uplift to produce enhanced convection on its forward and/or western flanks. This will likely help increase the storm translation speed, as PV generation by convection will constructively combine with advection to increase the PV tendency (Chang 1982). After landfall, however, the asymmetric convection may shift toward the storm's rear flank as the circulation center passes over the CMR. This may act to reduce the storm's forward motion, increasing its residence time over the island. In that event, a positive feedback may exist between storm motion and rainfall in which diabatic heating associated with the convection causes the storm to linger longer over the island, enhancing and prolonging the rainfall and convective activity that is serving to slow the storm motion.
This paper examines the effect of the topographically phase-locked convection on the geographic asymmetry of typhoon motion crossing the island of Taiwan. Observations are presented in section 2. Section 3 describes the Weather Research and Forecasting model (WRF) numerical experiment and the PV tendency diagnosis. The WRF simulations and the PV tendency diagnoses are presented in section 4 . Section 5 summarizes the results.

\section{Observations}

In addition to the 61 continuous-track cases in the 1960-2010 database, a further 23 typhoons exhibited discontinuous tracks (labeled DIS), owing to secondary low development after making landfall at Taiwan's eastern coast. Hourly typhoon center positions for the 84 events were collected from the CWB typhoon database (Wang 1980; Shieh et al. 1998). Radar and satellite information were employed to help refine the landfall/ departure positions and times of typhoons from 1977 onward. Intensity is defined as the typhoon's maximum wind recorded when the center was within $1^{\circ}(111.1 \mathrm{~km})$ of Taiwan. From landfall/departure positions and times, an overland translation speed (linear distance divided by duration time) for each typhoon can be estimated. ${ }^{1}$ Some statistical data are reported in Table 1.

Track discontinuities associated with surface secondary low development often result in exceptionally fast translation speeds over land $\left(18.7 \mathrm{~m} \mathrm{~s}^{-1}\right.$ on average, with the maximum value exceeding $61 \mathrm{~m} \mathrm{~s}^{-1}$ ). As these cases are clearly outliers, we defined our "slow motion," "medium motion," and "fast motion" groups with respect to the 61 continuous-track typhoons, which had a mean speed of $6.2 \mathrm{~m} \mathrm{~s}^{-1}$ while over land. Slow and fast storms are those with translation speeds one standard deviation $\left(2.9 \mathrm{~m} \mathrm{~s}^{-1}\right)$ smaller and larger than the continuous-track group mean (i.e., $3.3 \mathrm{~m} \mathrm{~s}^{-1}$ and $9.1 \mathrm{~m} \mathrm{~s}^{-1}$, respectively). If these criteria were then applied to the 23 discontinuous cases, then 17 (or $74 \%$ ) would belong to the fast group, 4 in the medium group, and 2 would be slow movers.

Figure 2 reveals that a significant geographic asymmetry exists with respect to continuous-track typhoon overland translation, with $77 \%$ (10 of 13 ) of the slowmoving storms making landfall on the northern segment of Taiwan's eastern coast and $60 \%$ (6 of 10) of the fast cases reaching the southern segment instead. This can

\footnotetext{
${ }^{1}$ In general, landfall positions are more precisely determined than departure points. Reflecting the higher quality of the data during and after 1997, we estimate the uncertainty of the departure point to be about $10-20 \mathrm{~km}$. Given the average overland period of $7-8 \mathrm{~h}$, this implies a translation speed uncertainty of about $1 \mathrm{~m} \mathrm{~s}^{-1}$, which we deem acceptable.
} 

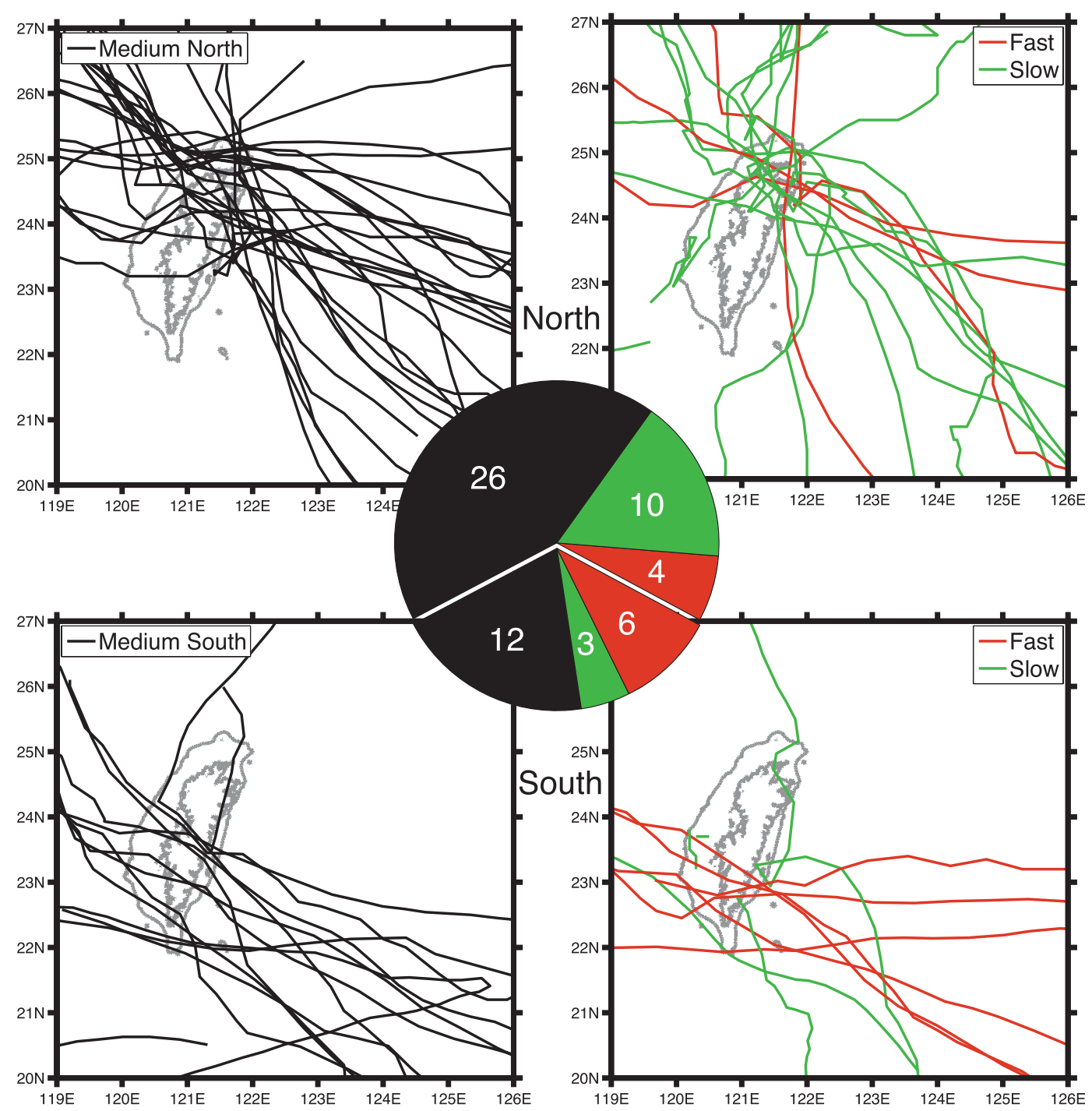

FIG. 2. Tracks of the continuous-track typhoons, subdivided by landfall latitude relative to $23.5^{\circ} \mathrm{N}$ and overland translation speed. Overlaid pie chart shows numbers and relative proportions of NLT and SLT cases-fast (red), medium (black), and slow (green) speed classes—based on overland translation speed.

profoundly influence the rainfall produced over Taiwan, as indicated by Fig. 3, which presents total rainfall during the overland period for the 21 CWB surface stations as a function of the translation speed, latitude, and maximum storm intensity. The figure emphasizes again that more of the slower typhoons are from the NLT class and are associated with larger precipitation totals. In contrast, typhoon intensity, as measured by maximum wind speed, does not appear to be a determining factor in precipitation production.

Table 1 reveals that both the mean crossing (overland) distance and mean translation speed of the SLT are longer than that of the NLT, while the average NLT storm spends 1.3 more hours over land and produces almost twice as much precipitation. The differences are not statistically significant, owing to large case-to-case variability, although the implications for Taiwan rainfall can be profound. The starkest difference is between the fast and slow TCs, which have mean translation speeds of 10.6 and $2.4 \mathrm{~m} \mathrm{~s}^{-1}$, respectively. The slow storms are over land 5 times longer and produce about 5.2 times more rain than their counterparts in the fast group.

Figure 4 shows 3 -hourly mean translation speed variations from $12 \mathrm{~h}$ before landfall (labeled "l") to $12 \mathrm{~h}$ after departure (labeled "d"), stratified by landfall latitude (Fig. 4a) or speed class (Fig. 4b). Note continuously tracking typhoons accelerate, on average, as they approach Taiwan's eastern coast, which has previously been noted (Yeh and Elsberry 1993a). While over land, however, the average NLT speed decreases, while their 


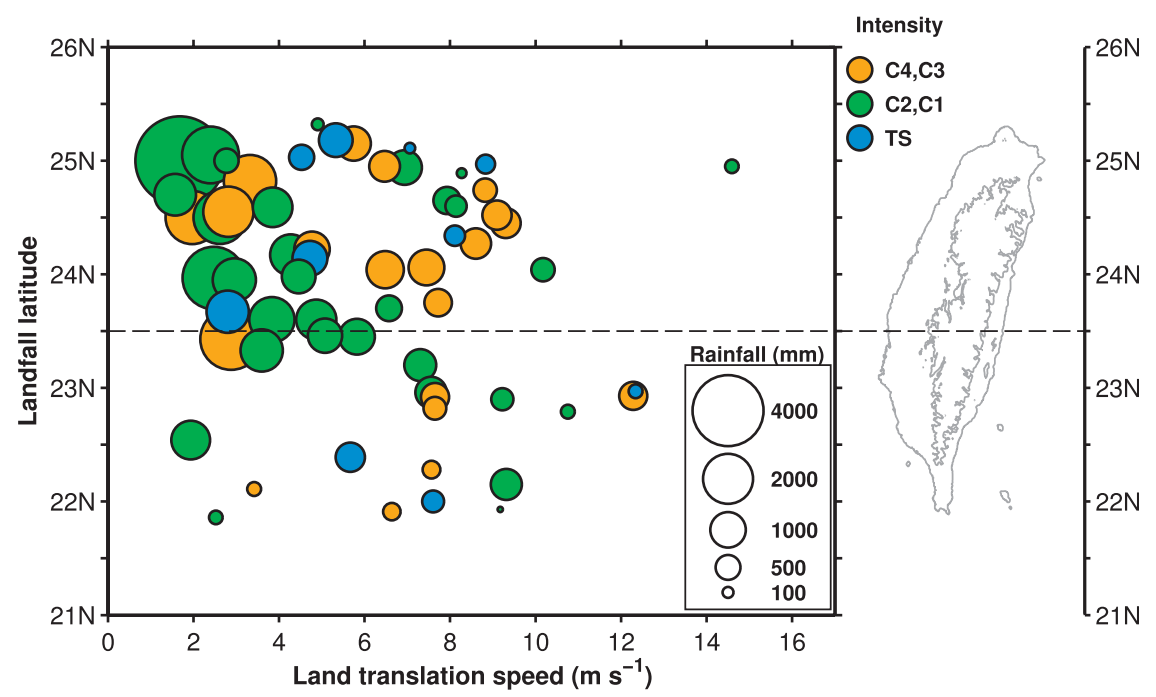

FIG. 3. The rainfall amount ( $\mathrm{mm})$ during landfall from $21 \mathrm{CWB}$ surface stations as a function of overland translation speed and landfall latitude for the 61 continuous-track typhoons. Rainfall amount is proportional to the area of circle. The color is the typhoon's intensity before landfall as represented by the Saffir-Simpson hurricane scale (categories 1-4 labeled as $\mathrm{C} 1-\mathrm{C} 4$, and TS is tropical storm strength).

SLT counterparts continue to speed up (Fig. 4a). As noted earlier, storms in the discontinuous group have a very large mean speed while over land, which is their primary distinguishing factor.

For Fig. 4b, the medium-speed class is further subdivided into northern and southern subgroups, again based on their landfall latitude relative to $23.5^{\circ} \mathrm{N}$. Among these TCs, the prelandfall speed increase is larger in the northern subgroup. After landfall, the slow movers and the medium north subgroup typhoons decelerate markedly. For comparison, we also show the temporal speed variations for typhoon cases that produced total rainfall amounts exceeding $1000 \mathrm{~mm}$ while over land. The relative postlandfall deceleration is comparable to the slow typhoons, which on average produced more than $2000 \mathrm{~mm}$ of precipitation after landfall (Table 1).

For all cases combined, the typhoon-produced rainfall tends to be largest on the western side of the CMR, at Alishan station, located near latitude $23.5^{\circ} \mathrm{N}$ (Fig. 5). ${ }^{2}$

\footnotetext{
${ }^{2}$ A recently developed climatology-based quantitative precipitation forecasting model for Taiwan (Lee et al. 2006; Cheung et al. 2008) incorporating hourly rainfall data from 319 CWB automatic rain gauges also reveals occasional large rainfall at the northern and southern tips of the CMR when typhoons are located northward of $23^{\circ} \mathrm{N}$. Some of these events involve typhoons passing by Taiwan without making landfall; that said, the lack of long-term surface station data at the southern tail and northern mountain area of CMR may cause rainfall underestimation in our analyses.
}

The peak is most pronounced in the slow, the medium north, and the NLT groups (Figs. 5a-c). The average rainfall at the Alishan station is 325 (162) mm for the slow (medium north) group typhoons, and the rainfall sum over all 21 surface stations is 2128 (809) $\mathrm{mm}$. None of the fast movers results in more than $50 \mathrm{~mm}$ of rain during the postlandfall period (Fig. 5d); however, even in those cases, the largest rainfall is received in the Alishan region, which means the typhoon rainfall pattern varies less than the totals. The pattern is thus phase locked with the topography, reflecting the interaction of the typhoon circulation and the CMR (Chang et al. 1993).

In summary, our analysis reveals a distinct geographic asymmetry with respect to typhoons that have slow and fast translation speeds across Taiwan. Most of the slow movers are NLT that decelerate after landfall, while the quickly translating storms are SLT that tend to exhibit postlandfall acceleration. The fast movers do not linger over the island long enough to produce large precipitation totals. The slow cases are prodigious producers of rainfall, which is maximized on the southwestern slope of the CMR.

Furthermore, the rainfall pattern is phase locked with the topography. Since diabatic heating associated with convection may affect the PV tendency, this phase locking may profoundly influence a typhoon's motion across the island, with an impact depending on where the terrain-induced convection is relative to the storm. We suspect that diabatic heating associated with convective 
(a)

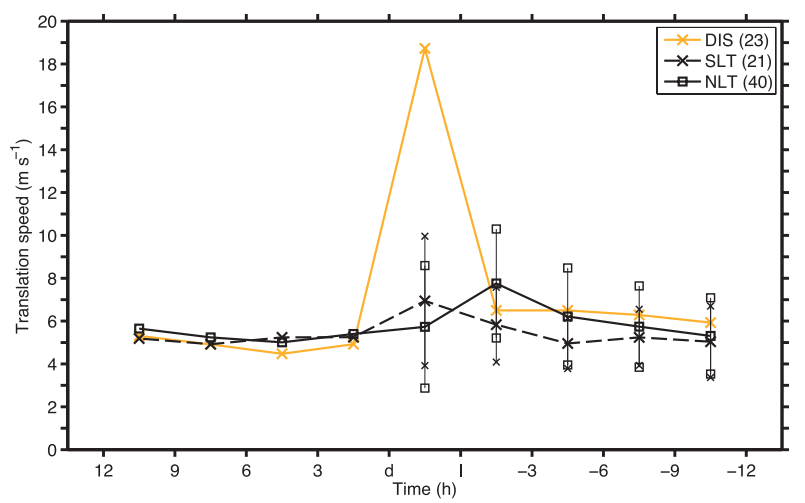

(b)

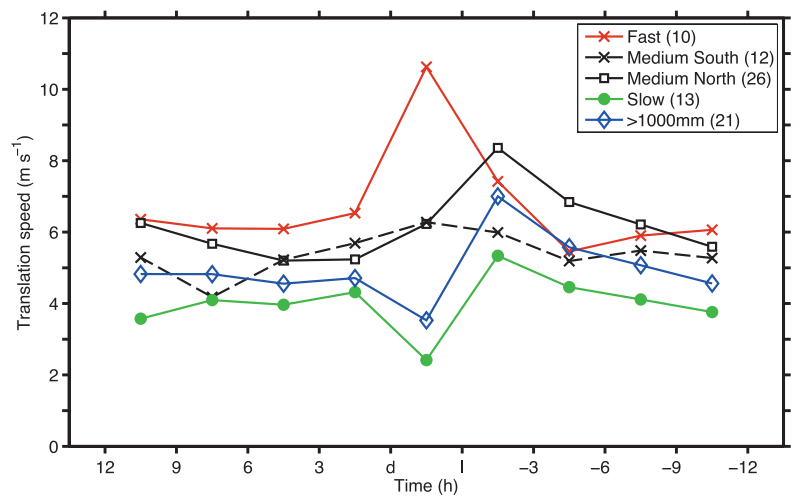

FIG. 4. The 3-hourly mean translation speeds from $12 \mathrm{~h}$ before landfall ("l") to $12 \mathrm{~h}$ after departure ("d") for the eastern coast landfall cases subdivided with respect to (a) landfall latitude, for continuous (NLT, SLT) and DIS cases, and one standard deviation before departure is also added for NLT and SLT; and (b) overland translation speed class (fast, medium north, medium south, and slow) for the continuous cases. In (b), the average of cases having CWB gauge rainfall amounts over $1000 \mathrm{~mm}$ while overland is also shown for comparison.

rainfall over the CMR is a principal factor in bringing about the reduced speeds - and large rainfalls — of the slow-moving NLT cases, and it can help explain the faster overland motions of the SLT and prelandfall accelerations as well. This is investigated with the assistance of semi-idealized simulations in the next section.

\section{Model settings and potential vorticity tendency diagnosis}

A "semi-idealized" variant of the "real data" WRF model (version 3.1.1) is used to investigate the speed variations of typhoons owing to topographically phaselocked convection. It is an aquaplanet WRF model based on the Advanced Research WRF (ARW) real data core. This variant has previously been used to study the impact of microphysics and cloud-radiative processes as well as model initialization on hurricane tracks (Fovell and $\mathrm{Su}$ 2007; Fovell et al. 2009, 2010; Cao et al. 2011). Model physics options selected include the Yonsei University (YSU) planetary boundary layer scheme, Lin et al. (1983) microphysics scheme, Rapid Radiative Transfer Model (RRTM; Mlawer et al. 1997) and Dudhia (1989) radiation packages, and the Smagorinsky option for subgrid mixing. The simulations employ a single-square domain, $1500 \mathrm{~km}$ on a side, and the model top is placed at $10 \mathrm{hPa}$. The horizontal resolution is $5 \mathrm{~km}$, and there are 35 model levels in the vertical.

For consistency with previous semi-idealized studies, the base state consists of Jordan's (1958) Caribbean hurricane-season composite temperature and humidity sounding with a uniform sea surface temperature (SST) of $29^{\circ} \mathrm{C}$. The simulations are initialized with easterly flow that is uniform in both the horizontal and vertical directions, with magnitudes that are varied among the experiments. The typhoons are established by inserting an artificial vortex, created using the WRF tropical cyclone bogus scheme, into the initial state. This scheme supplies the vortex a modified Rankine wind profile beyond the radius of maximum wind that is held fixed between the surface and $600 \mathrm{hPa}$, and is linearly decreased to nearly zero by $100 \mathrm{hPa}$.

The model domain has no topography other than the island of Taiwan, which was constructed using the 30-s-resolution U.S. Geological Survey (USGS) database. Previous studies have suggested that the land-sea contrast by itself will induce asymmetric convection and affect the typhoon track as it approaches landfall (Tuleya and Kurihara 1978; Tuleya et al. 1984; Wong and Chan 2006, 2007). Therefore, to focus more closely on the direct terrain effect, we have given our Taiwan grid points the same roughness length and moisture availability as the ocean. As a consequence, our Taiwan is an island made of water. While technically there is no "landfall" or "overland" period in these simulations, we will still use these terms to describe the encounter of the model storms with the Taiwan topography.

To analyze the motion of our tropical cyclones, we employ the diagnostic PV tendency method (WW00; WW01) and inviscid PV tendency equation in the following form:

$$
\frac{\partial P}{\partial t}=-\mathbf{V} \cdot \nabla_{h} P-w \frac{\partial P}{\partial z}+\rho^{-1} \nabla_{3} \cdot(Q \mathbf{q}),
$$

where $P$ is $\mathbf{P V}, \mathbf{V}$ is horizontal wind, $\nabla_{h}$ is the horizontal gradient operator, $w$ is vertical velocity, $\rho$ is density, $Q$ is diabatic heating, $\mathbf{q}$ is the absolute vorticity vector, 

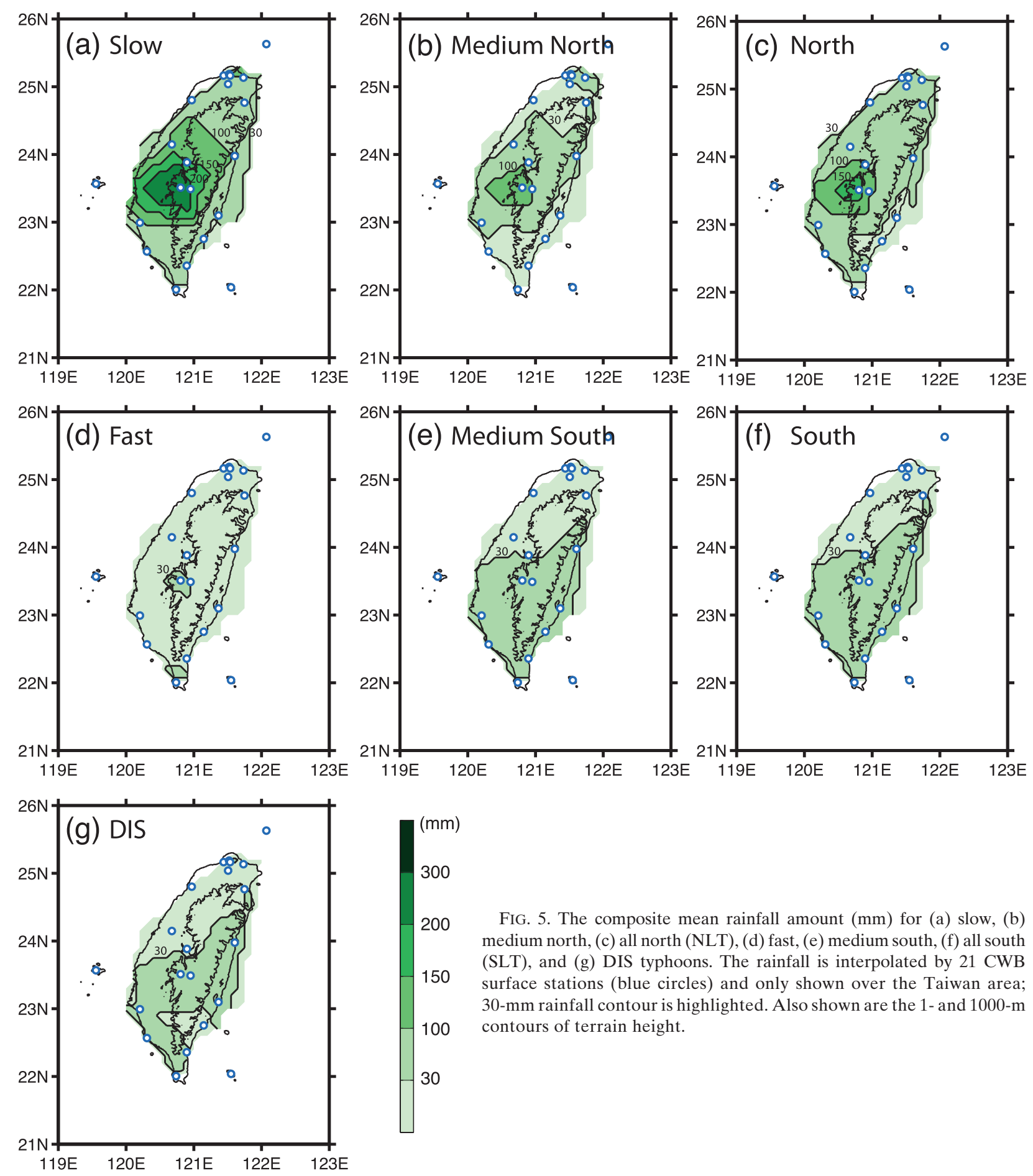

FIG. 5. The composite mean rainfall amount (mm) for (a) slow, (b) medium north, (c) all north (NLT), (d) fast, (e) medium south, (f) all south (SLT), and (g) DIS typhoons. The rainfall is interpolated by $21 \mathrm{CWB}$ surface stations (blue circles) and only shown over the Taiwan area; $30-\mathrm{mm}$ rainfall contour is highlighted. Also shown are the 1- and 1000-m contours of terrain height.

and $\nabla_{3}$ is the three-dimensional gradient operator. The net PV tendency is determined by three terms on the right-hand side of (1): horizontal advection (HA), $\mathrm{DH}=\frac{1}{\rho}\left[(\zeta+f) \frac{\partial Q}{\partial z}+\left(\frac{\partial u}{\partial z}-\frac{\partial w}{\partial x}\right) \frac{\partial Q}{\partial y}+\left(\frac{\partial w}{\partial y}-\frac{\partial v}{\partial z}\right) \frac{\partial Q}{\partial x}\right]$ vertical advection (VA), and the diabatic heating (DH) term that depends on gradients of $Q$ and q. The $\mathrm{DH}$ term can be expanded as

where $\zeta$ and $f$ are relative and planetary vorticity, respectively. Our results suggest that the DH term is 
dominated by the term involving the vertical gradient of $Q$.

WW00 and WW01 presume that a TC dominated by its symmetric circulation moves toward the maximum in the azimuthal wavenumber-1 (WN1) portion of PV tendency in a fixed frame, expressed as

$$
\left(\frac{\partial P}{\partial t}\right)_{1}=-C_{x} \frac{\partial P_{s}}{\partial x}-C_{y} \frac{\partial P_{s}}{\partial y}
$$

where $P_{s}$ is the symmetric part of the vortex $\mathrm{PV}$, the subscript " 1 " denotes the WN1 component, and $C_{x}$ and $C_{y}$ are scalar speeds in the east and north directions, respectively. The left-hand side of (3) represents the net PV tendency, representing storm motion or the contribution to the PV tendency from each term (HA, VA, $\mathrm{DH})$ from the right-hand side of (1), separately or in combination. The speeds $C_{x}$ and $C_{y}$, which are determined via least squares as in WW00 and WW01, form a vector representing the magnitude and direction of the storm motion or the component contributions. For a symmetric vortex, the horizontal gradients $\partial P_{s} / \partial x$ and $\partial P_{s} / \partial y$ both have zero mean and are also uncorrelated, so the scalar speeds given by

$$
\begin{gathered}
C_{x}=-\frac{\sum_{i=1}^{N} \overline{\left(\frac{\partial P_{s}}{\partial x}\right)_{i}} \overline{\left(\frac{\partial P}{\partial t}\right)_{1 i}}}{\sum_{i=1}^{N} \overline{\left(\frac{\partial P_{s}}{\partial x}\right)_{i}^{2}}} \\
C_{y}=-\frac{\sum_{i=1}^{N} \overline{\left(\frac{\partial P_{s}}{\partial y}\right)_{i}} \overline{\left(\frac{\partial P}{\partial t}\right)_{1 i}}}{\sum_{i=1}^{N} \overline{\left(\frac{\partial P_{s}}{\partial y}\right)_{i}^{2}}},
\end{gathered}
$$

are completely independent. These equations also imply that a component like DH influences the motion most where the PV gradients are largest (at the edge of the symmetric vortex) and that the TC's motion is not influenced by distant heating, at least not directly.

We believe the PV tendency method can help us understand how and why asymmetric convection associated with terrain influences TC motion. However, one significant limitation of the method (demonstrated in Fig. 11 of WW00) is that the component vectors canand do-change amplitude and shift orientation with height, in both absolute terms and relative to each other, even when the vortex translation is uniform with altitude. Another limitation is that terms like HA, VA, and DH are not independent, and they strongly modulate each other (Cao et al. 2011). In this application, HA and
VA incorporate not only beta drift (e.g., Holland 1983) but also the purely physical influences of the obstacle on the flow, such as lifting, sinking, blocking, and channeling (e.g., Kuo et al. 2001; Lin et al. 2005; Jian and Wu 2008). Yet, the physical disturbances caused by topography also provoke alterations in convective heating, reflected in $\mathrm{DH}$, that further influence the airflow encountering the terrain. These characteristics make it difficult to employ the method in a strictly objective fashion and perhaps impossible to separate the purely physical aspects of the interaction with topography from the diabatic effects.

In this paper, we are primarily interested in the role of diabatic heating on TC motion, and the analysis for the most part will emphasize qualitative comparisons, such as between simulations or between different time periods for the same simulation. To minimize the issue of height-dependent component orientation, we average the DH term over an appreciable depth [from 1 to $11 \mathrm{~km}$ above ground level, represented by the overbars in (4) and (5)]. This averaging layer depth was selected empirically.

\section{Numerical results}

\section{a. Overview of the $O C$ and T3N experiments}

Figure 6 compares 3-hourly tracks from a simulation using real Taiwan topography (run T3N) with an oceanic control (run OC) case lacking topography. Both commenced with identical bogussed typhoons having a $50 \mathrm{~m} \mathrm{~s}^{-1}$ maximum wind $V_{m}$ at $50-\mathrm{km}$ radius from the center (radius of maximum wind $R_{m}$ ), embedded in a $3 \mathrm{~m} \mathrm{~s}^{-1}$ uniform easterly mean flow. The typhoons are initially placed at $20.5^{\circ} \mathrm{N}, 124.5^{\circ} \mathrm{E}$, which is $400 \mathrm{~km}$ east of Taiwan for the T3N run and results in a landfall on the northern ("N") segment of Taiwan's eastern coast; hence, the experimental designation "T3N." The shading is the total rainfall difference between the T3N and OC experiments through $72 \mathrm{~h}$ of model integration.

In both experiments, the storms generally move toward the northwest due to the steering flow and the $\beta$ effect. However, significant differences emerge as the T3N storm approaches Taiwan. The OC storm's average motion between hours 27 and 36 is $4.0 \mathrm{~m} \mathrm{~s}^{-1}$, although with some speed and direction fluctuations as suggested by Fig. 6 . In contrast, the T3N typhoon's speed increases from about $4.0 \mathrm{~m} \mathrm{~s}^{-1}$ (between 27 and $30 \mathrm{~h}$ ) to $4.2 \mathrm{~m} \mathrm{~s}^{-1}$ (for hours 30-33), finally reaching $5.2 \mathrm{~m} \mathrm{~s}^{-1}$ in the $2 \mathrm{~h}$ before landfall at $35 \mathrm{~h}$. This is quite consistent with the result of the NLT composite (Fig. 4a).

Note further that the T3N experiment's TC turns sharply westward, toward the island, during the prelandfall 


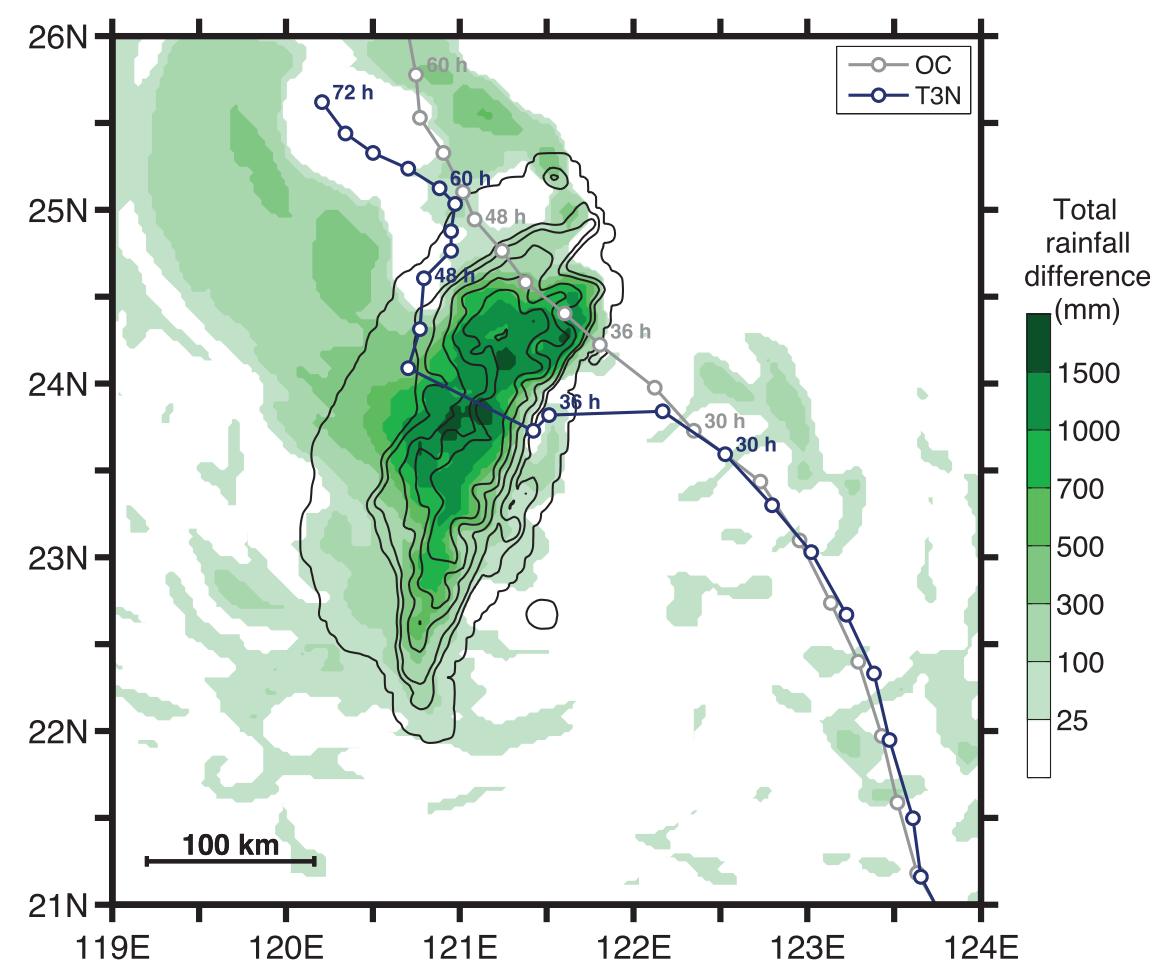

FIG. 6. T3N (blue) and OC (gray) experiments with initial $3 \mathrm{~m} \mathrm{~s}^{-1}$ easterly mean flow. Track positions are defined by the local surface wind minimum near the sea level pressure minimum. Shading is the total rainfall difference, T3N - OC, over the 72-h simulation period. Only part of the $1500 \mathrm{~km}^{2}$ domain is shown.

acceleration after $33 \mathrm{~h}$. Although the observations reveal a great diversity of tracks among NLT storms (Fig. 2), it remains there are numerous examples of typhoons that made landfall along the northern segment of Taiwan's eastern coast following a westward deflection from their earlier, generally more northwestern motions. While there is some ambiguity involved in identifying the surface circulation center when the storm is over the steep CMR, once clearly located west of the ridge the T3N storm moves northward along the island's northwestern coast, parallel to the CMR, and slows progressively along the way (Fig. 6). Between hours 45 and 48 , for example, the storm motion is $3.0 \mathrm{~m} \mathrm{~s}^{-1}$, but this decreases to 2.4 and $1.2 \mathrm{~m} \mathrm{~s}^{-1}$ in the next two 3-hourly periods, respectively. The T3N typhoon spends $22 \mathrm{~h}$ (from 35 to $57 \mathrm{~h}$ ) over the island and travels $146.2 \mathrm{~km}$ between landfall and departure, making an average overland motion of $1.8 \mathrm{~m} \mathrm{~s}^{-1}$. The TC remains a slow mover relative to its OC counterpart long after its center moves past the island (out to $72 \mathrm{~h}$ ).

Topographic lifting and substantially slowed motion combine to produce copious amounts of precipitation over the Taiwan area. We estimate the rainfall amount by averaging the gridded rainfall within $10 \mathrm{~km}$ of the locations of the $21 \mathrm{CWB}$ surface stations. The total rainfall in the T3N experiment is $5437 \mathrm{~mm}$ during the 22-h overland period, which is much larger than the OC storm produced during the same time interval $(3887 \mathrm{~mm}){ }^{3}$ Most of the rainfall variations emerge after landfall. During the overland period, the rainfall difference mainly occurs over the CMR. Subsequent rainfall differences may be due to the storm-track and structure differences.

\section{b. Motion of the OC and T3N typhoons}

When the $\mathrm{T} 3 \mathrm{~N}$ cyclone is relatively far from the island in terms of distance and time, there is little difference between it and its OC counterpart in terms of structure, motion, and position. During this period, the PV equation's DH term is small and typically directed toward the rear flank, thereby representing a minor negative influence on the storm speed (see prior to $30 \mathrm{~h}$ in Fig. 7). This is because the storms possess a high degree of

\footnotetext{
${ }^{3}$ The rainfall amount reported in Table 2 for the OC case is smaller, as it reflects the shorter period of time in which that storm resides in the area occupied by Taiwan.
} 


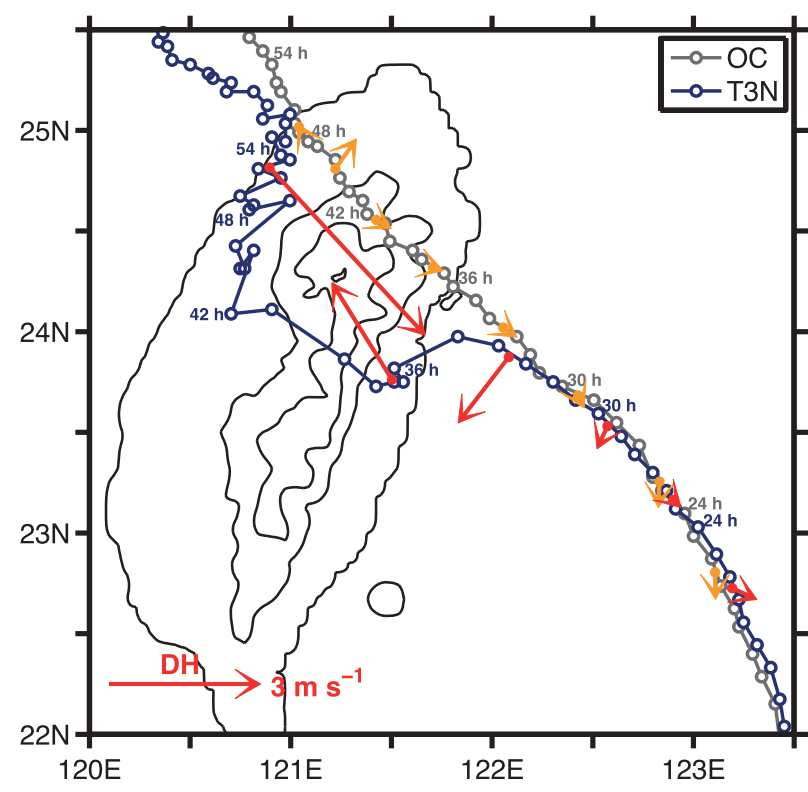

FIG. 7. As in Fig. 6, but depicting the variation of the DH contribution to storm motion (vectors) at various times for the $\mathrm{OC}$ (orange) and $\mathrm{T} 3 \mathrm{~N}$ (red) experiments. Averaging period for each vector is $4 \mathrm{~h}$, except for the 48-57-h period of run T3N. This time period is chosen in order to examine the slow TC motion along the Taiwan western coast.

azimuthal symmetry, as suggested by the fields for T3N (Figs. 8a,b). The TCs are spatially compact with circular flow (Fig. 8b), with moderate amounts of columnaveraged diabatic heating (Fig. 8a) and rainfall (Fig. 8b). In Fig. 8a, the blue contours depict the symmetric PV structure used in the calculation of the DH term (3), and this can be used as a proxy for storm size, intensity, and structure.

The $\mathrm{T} 3 \mathrm{~N}$ storm, however, becomes progressively more asymmetric as it approaches the island, and this is reflected in the large but time-dependent influence of the DH term on the motion seen in Figs. 7 and 8. After $30 \mathrm{~h}$ but before landfall, the developing WN1 asymmetry in DH appears to account for the TC's southwestward deflection relative to the OC case (Fig. 7). The $Q$ and rainfall fields have maxima on the storm's northern flank (Figs. 8c,d). DH has a component in the direction of storm motion during this time, which means it is helping to produce the previously noted acceleration during the approach to landfall.

Once inland (around $36 \mathrm{~h}$ ), but before passing over the CMR, the DH term remains large but shifts toward the CMR. The storm clearly has difficulty surmounting the steep terrain (Fig. 7). After passage, DH still points toward the CMR, which is to the southeast of the now-enlarged cyclone center (Fig. 8e). During this time, the storm moves very slowly (Fig. 7), consistent with the large contribution of $\mathrm{DH}$ against the beta drift and large-scale steering. These variations in $\mathrm{DH}$ are clearly due to the impact of topography on convection, as the $\mathrm{DH}$ contribution to motion remains small in the OC case, apart from occasional, relatively unimportant outbreaks of somewhat more vigorous convection (not shown).

A curious aspect of the southwestward deflection period between 32 and $35 \mathrm{~h}$ is that the DH term is clearly pointing toward a local minimum in convective heating and rainfall (Figs. 8c,d). The presence and location of the minimum makes good sense: part of the cyclone's circulation is passing over land, and the convective activity is diminished where that flow is effectively directed downslope. We need to recall that the DH term depends not on $Q$, but rather on its gradients, and specifically on the WN1 component. In a cyclonic vortex, $\mathrm{PV}$ is being created where diabatic heating increases with height.

Figure 9 presents a vertical cross section of the $Q$ and DH WN1 fields at this time, oriented southwestnortheast across the storm center and along the $\mathrm{DH}$ vector shown on Fig. 8c. Convection is clearly deeper and stronger in the northeast quadrant, but the heating maximum is in the lower troposphere, leading to negative vertical gradients through much of the troposphere. On the southwestern side, convection is weaker and less organized, and nearly absent at the radial position corresponding to the northeastern flank eyewall (cf. near -30 and $+30 \mathrm{~km}$ ). DH's WN1 component necessarily depends on the heating structure at azimuths and radii not shown here, but it remains that the negative values in the northeast quadrant and near-zero gradients to the southwest at the same radius combine to put a relative maximum of WN1 DH on the southwestern side when averaged over the free troposphere, as indicated by the $\mathrm{DH}$ vector. This may seem odd, but the bottom line is that the DH term is encouraging southward and westward motion in the T3N storm relative to the undisturbed OC case, during a period when the T3N storm shifts in precisely that way.

During the 48-57-h period, in which the T3N typhoon is moving very slowly northward along the island's western coast, there are two regions of particularly large $Q$, on the southern and eastern flanks of the storm (Fig. $8 \mathrm{e})$. Both are associated with upslope flow, as suggested by the low-level composite circulation vectors (Fig. 8f). Note that the enlarged TC straddles the CMR at this time, so the southern maximum occurs where the TC's southwesterly winds are being forced to ascend up the western side of the CMR, whereas the eastern flank ascent occurs on the east-facing slopes. In between, there is a prominent gap in the $Q$ field, which is located 
(a)

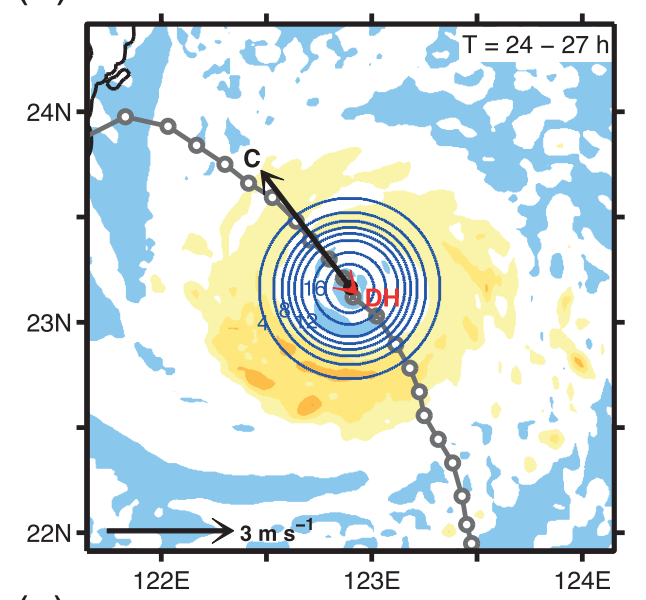

(c)

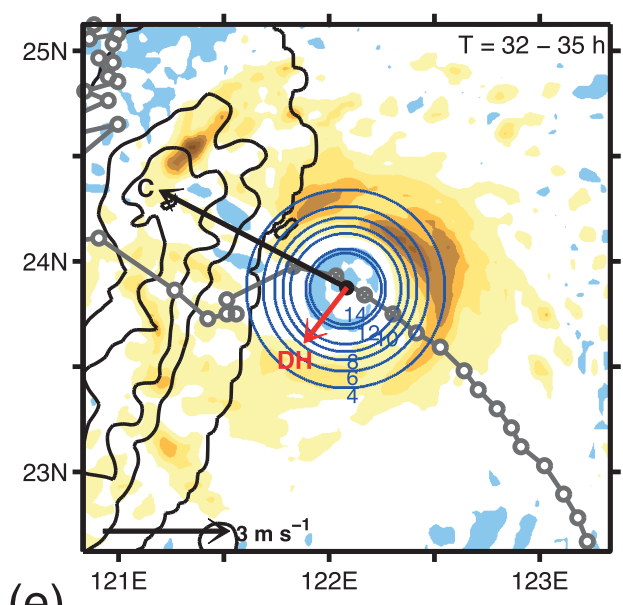

(e)

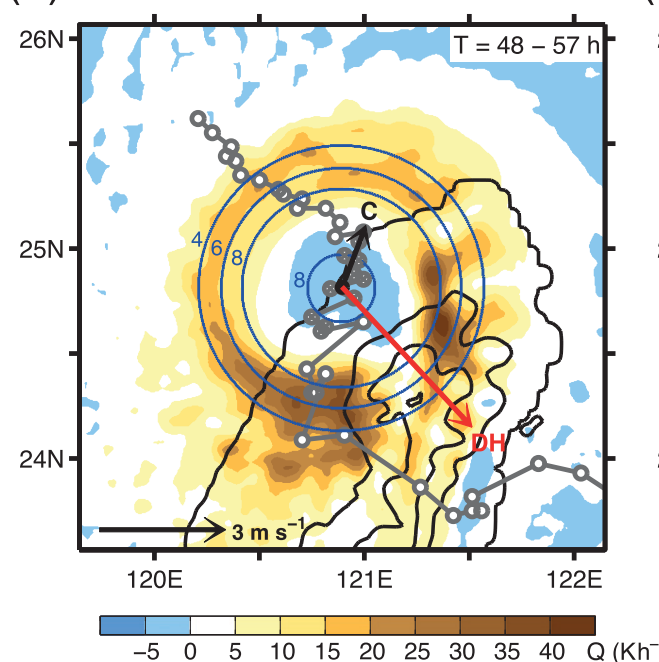

(b)

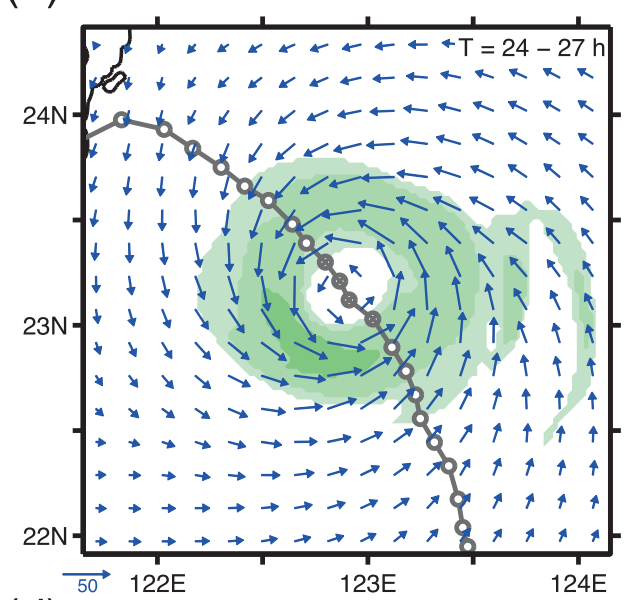

(d)

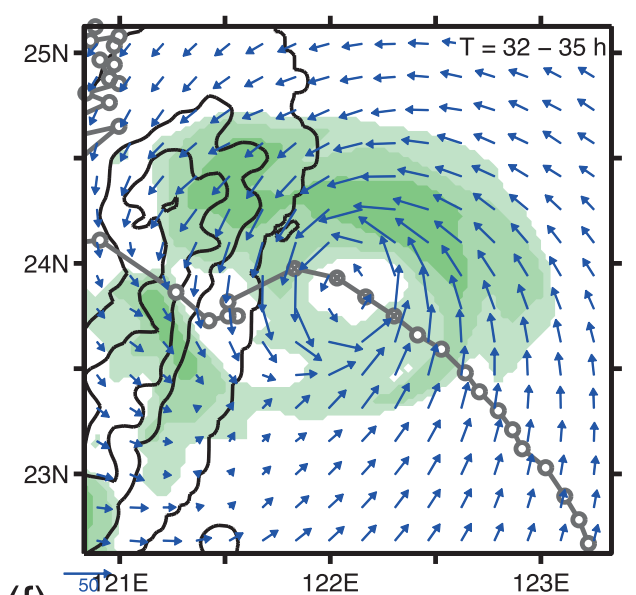

(f)

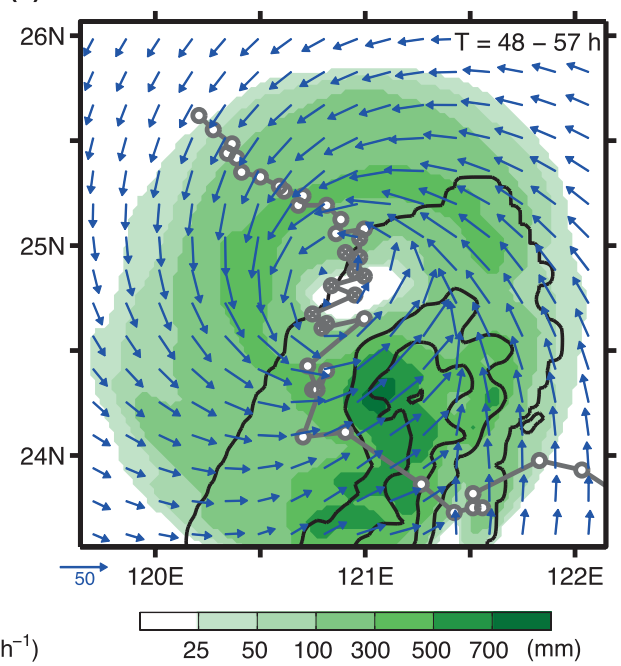

FIG. 8. Vortex-following composite fields for three different time periods from the T3N experiment. (a),(c),(e) Vertically averaged $Q$ (color shaded) and symmetric PV structure [blue contours, PVU (1 PVU $\left.=10^{-6} \mathrm{~m}^{2} \mathrm{~s}^{-1} \mathrm{~K} \mathrm{~kg}^{-1}\right)$ ], along with typhoon motion vector $\mathrm{C}$ (black), and the vector representing the contribution of the PV equation's DH term (red). (b),(d),(f) Composite rainfall (shaded) and wind vectors at the lowest level. Averaging periods are (a),(b) 24-27, (c),(d) 32-35, and (e),(f) 48-57 h, and represented by filled circles on the superimposed storm tracks. 


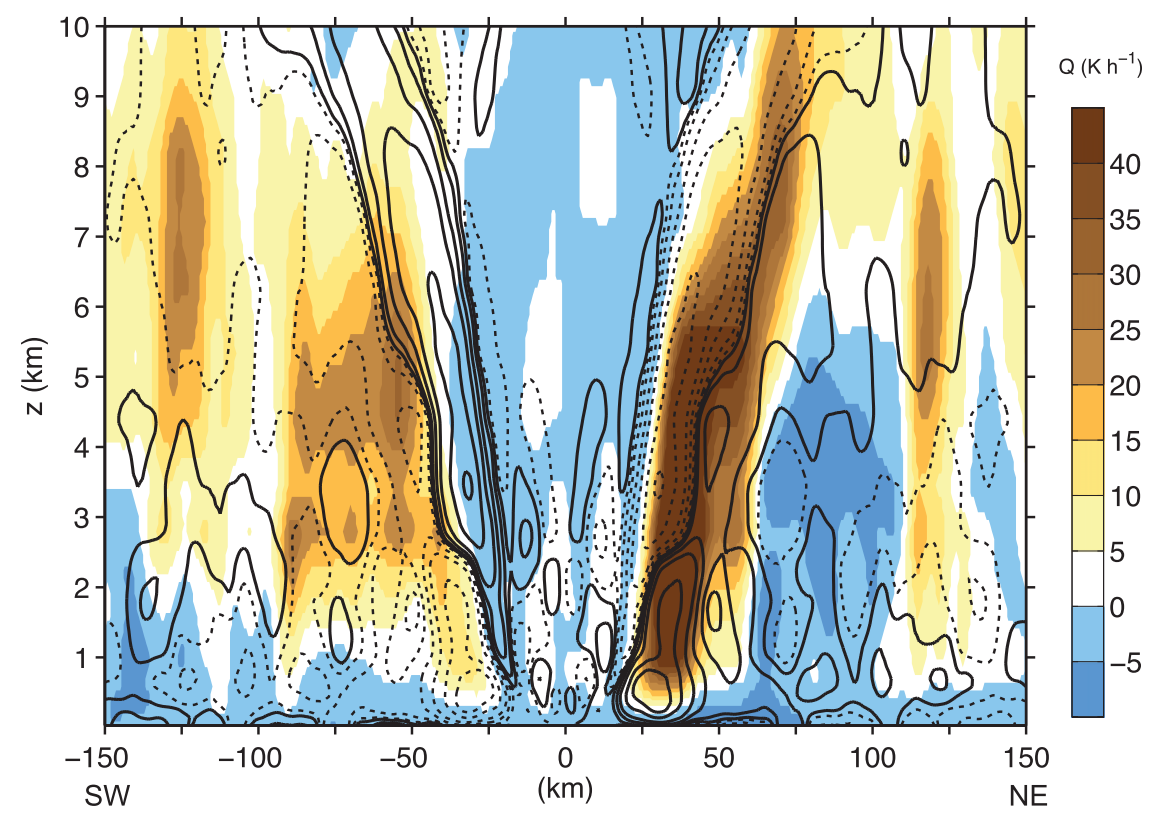

FIG. 9. Southwest-northeast cross section of $Q$ (color shaded) averaged between 32 and $35 \mathrm{~h}$ of T3N experiment (see Fig. 8c). Superposed is the WN1 component of the DH term (contoured at $1,5,15,30,60,90 \times 10^{-4} \mathrm{PVU} \mathrm{s}^{-1}$; negative values dashed).

where the TC's southwesterly flow passes the CMR's highest point and starts moving downslope (Fig. 8f).

The $\mathrm{DH}$ vector points toward that prominent gap, but this is partly an illusion and partly a consequence of the WN1 decomposition. The main point is that the DH term is powerfully opposing the beta drift and largescale steering, impacting speed and direction, both of which are important. The reduced speed that occurs when DH shifts to the storm's rear flank prevents the storm from moving quickly away from land. The component to the storm's right keeps it traveling along the coastline parallel to the CMR. Thus, the topographically phase-locked convection causes the TC to linger much longer over the island, permitting it to produce impressively large rainfall totals.

\section{c. Sensitivity tests}

Figure 10 presents variants of the Taiwan topography experiment in which the initial mean easterly current is strengthened to either 5 or $10 \mathrm{~m} \mathrm{~s}^{-1}$ (T5N and T10N, respectively). During the overland period, the mean storm translation speeds are 5.3 and $10 \mathrm{~m} \mathrm{~s}^{-1}$, respectively, far larger than the original T3N case. These storms spend less time over the island, produce much less rainfall (see Table 2), and their diabatic heating fields are both smaller and project less strongly onto the crucial WN1 component, as revealed by the smaller magnitudes of the $\mathrm{DH}$ vectors (relative to their own translation speeds and compared to the T3N case in Fig. 8e). Our interpretation is that asymmetric diabatic heating is less capable of slowing down typhoons that are strongly steered. Conversely, we see that reducing the steering to a critical point might bring about further slowing through the action of asymmetric convection.

We also examined the sensitivity to the initial size and strength of the vortex. Figure 11 shows the tracks and accumulated rainfall fields from two very similar simulations (T3V65 and T3R75) that result when an initially stronger $\left(V_{m}=65 \mathrm{~m} \mathrm{~s}^{-1}\right)$ or larger $\left(R_{m}=75 \mathrm{~km}\right)$ vortex is placed in the same base state used for the T3N and OC runs. The time period shown corresponds to when the storms are over land, west of the CMR. Like the T3N case, these TCs accelerate (and curve westward) as they approach the island's eastern coast; however, they are faster movers-both before and after landfall (Table 2). As a consequence, they spend less time over the island and produce less rainfall, although the rainfall remains concentrated on the rear flank and the $\mathrm{DH}$ contribution acts to substantially slow and alter storm motion, as it did in the T3N case.

Figure 12 shows the results of an experiment with an initially weaker cyclone $\left(V_{m}=25 \mathrm{~m} \mathrm{~s}^{-1}\right)$. Like the original T3N storm, this case turns westward and accelerates toward the island prior to landfall, and it exhibits substantially slower motion during the overland and postdeparture time periods (Table 2). Unlike the T3N typhoon, however, this storm is more compact (cf. symmetric PV field with Fig. 8b) and does not move 
(a)

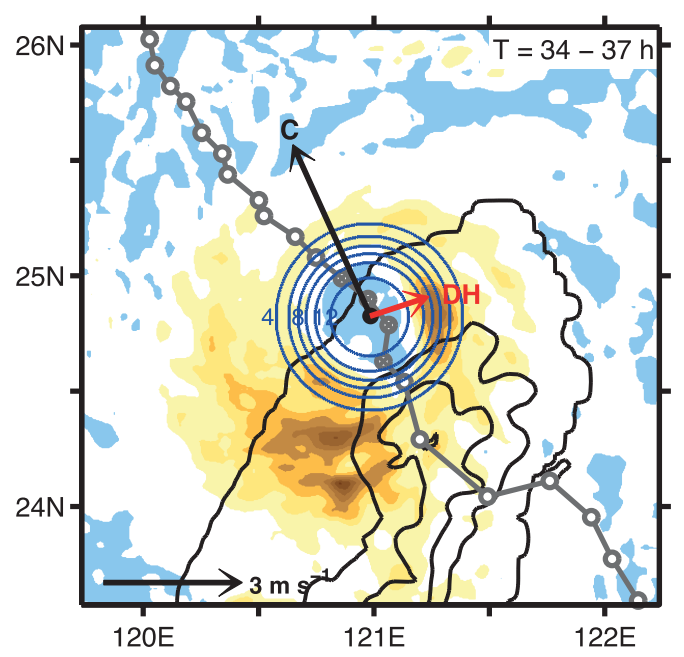

(c)

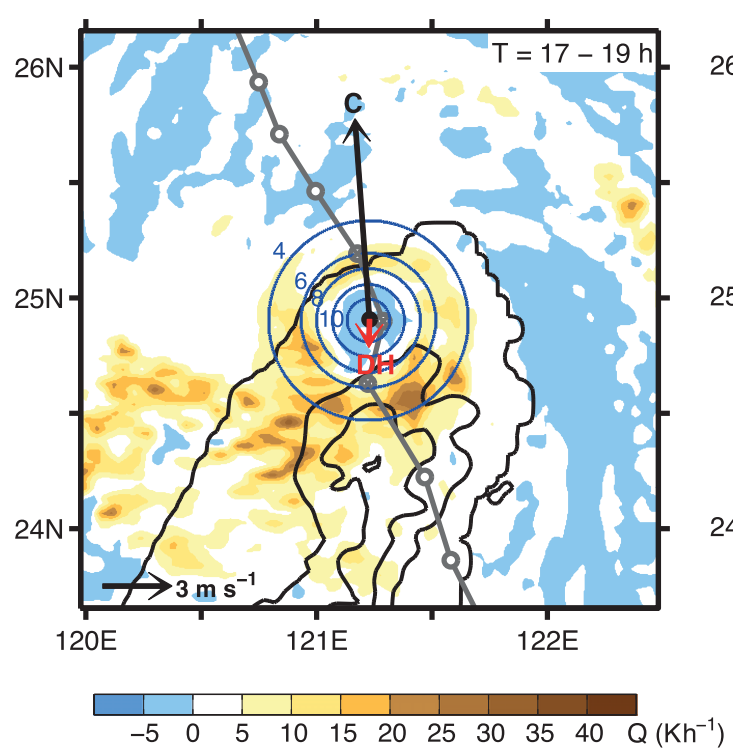

(b)

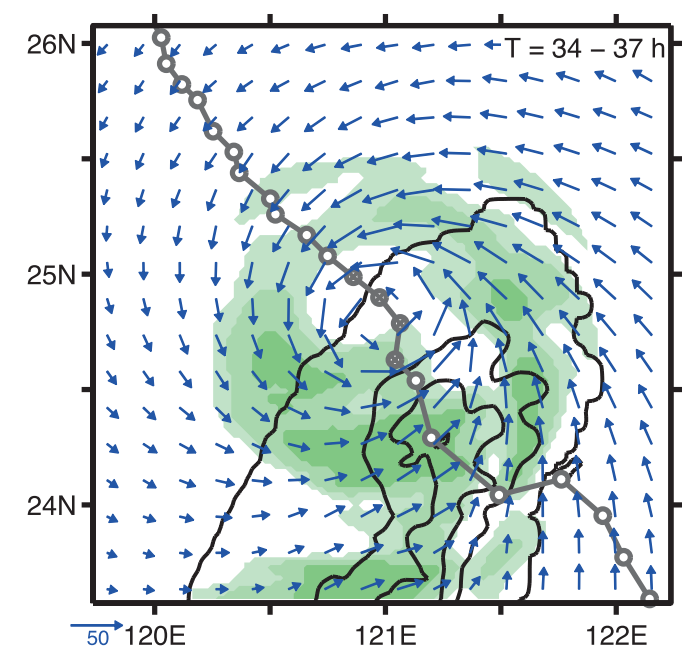

(d)

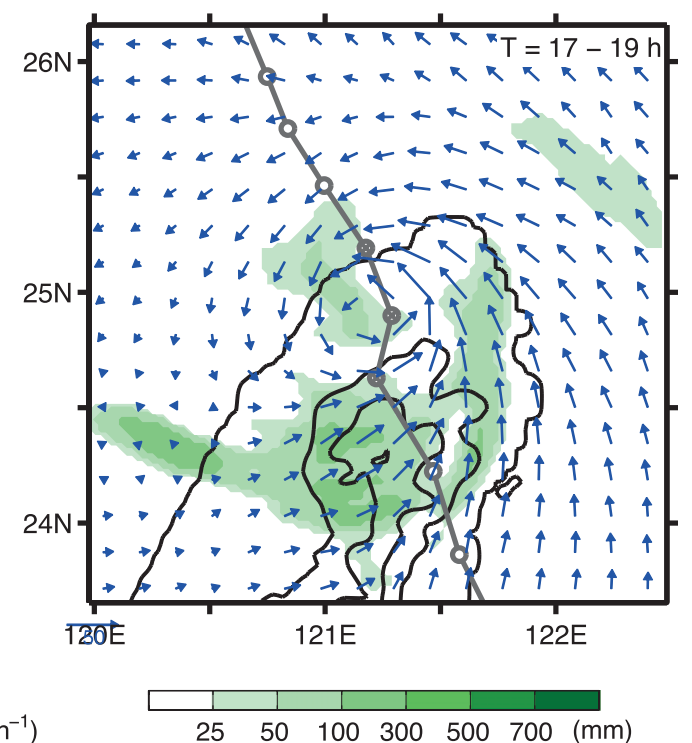

FIG. 10. As in Fig. 8, but for experiments with (a),(b) 5 and (c),(d) $10 \mathrm{~m} \mathrm{~s}^{-1}$ initial mean easterly currents and landfalls north of $23.5^{\circ} \mathrm{N}$ (experiments T5N and T10N).

along the western coast parallel to the CMR. Instead, its extended overland period comes from exceptionally slow motion while west of the ridge, a consequence of asymmetric diabatic heating associated with topography as indicated by the $\mathrm{DH}$ term. Despite this, rainfall during the overland period is relatively small $(1450 \mathrm{~mm})$, reflecting the TC's small size.

It can be anticipated that, depending on its angle of approach and latitude of landfall, PV generation by asymmetric diabatic heating may also help hasten the passage of a typhoon across the island. In case T5S, shown in Fig. 13, the combination of a $5 \mathrm{~m} \mathrm{~s}^{-1}$ initial easterly wind and a more equatorward vortex placement creates a landfall south of $23.5^{\circ} \mathrm{N}$ and a relatively short overland duration (Table 2). The no-island version of this simulation translated northwestward at about $5.3 \mathrm{~m} \mathrm{~s}^{-1}$ during this time (not shown). Storm T5S, in contrast, averaged $9 \mathrm{~m} \mathrm{~s}^{-1}$ between hours 30 and $35,{ }^{4}$ and executed an abrupt turn to the north upon passing the CMR.

\footnotetext{
${ }^{4}$ The $7.6 \mathrm{~m} \mathrm{~s}^{-1}$ value in Table 2 reflects the complete 8 -h overland period spanning $28-36 \mathrm{~h}$, inclusive.
} 
TABLE 2. Rainfall and translation data for some simulated typhoons. Translation speeds represent stages during which the storm center was 300-100 km away from the coast, between $100 \mathrm{~km}$ away and landfall, and while overland. The asterisk means that the statistics are computed relative to the spatial region occupied by Taiwan, which was not present in the simulation.

\begin{tabular}{|c|c|c|c|c|c|}
\hline Name & $\begin{array}{l}\text { 300-100-km translation } \\
\text { speed }\left(\mathrm{m} \mathrm{s}^{-1}\right)\end{array}$ & $\begin{array}{l}\text { 100-km-landfall translation } \\
\text { speed }\left(\mathrm{m} \mathrm{s}^{-1}\right)\end{array}$ & $\begin{array}{l}\text { Overland translation } \\
\text { speed }\left(\mathrm{m} \mathrm{s}^{-1}\right)\end{array}$ & $\begin{array}{c}\text { Overland } \\
\text { duration (h) }\end{array}$ & $\begin{array}{l}\text { Overland rainfall } \\
\text { amount }(\mathrm{mm})\end{array}$ \\
\hline $\mathrm{OC}^{*}$ & 4.1 & 3.9 & 2.5 & 17 & 2862.1 \\
\hline $\mathrm{T} 3 \mathrm{~N}$ & 3.9 & 4.6 & 1.8 & 22 & 5437.2 \\
\hline T3V65 & 4.3 & 4.5 & 2.6 & 17 & 4708.4 \\
\hline T3V25 & 2.9 & 3.6 & 3.8 & 11 & 1449.5 \\
\hline T3R75 & 4.5 & 3.1 & 3.0 & 15 & 4809.1 \\
\hline $\mathrm{T} 5 \mathrm{~N}$ & 4.9 & 5.8 & 5.3 & 7 & 1086.6 \\
\hline $\mathrm{T} 10 \mathrm{~N}$ & 9.4 & 8.1 & 10.0 & 5 & 764.6 \\
\hline T5S & 5.4 & 4.4 & 7.6 & 8 & 1017.7 \\
\hline
\end{tabular}

While over the island itself, the DH contribution as determined by the PV analysis is qualitatively consistent with T5S's northward turn after making landfall prior to $30 \mathrm{~h}$ and before departing shortly after $35 \mathrm{~h}$. Once past the island's western coast, the translation speed slows considerably and the storm reacquires a more northwestward track, as DH reverts to playing a minor role in the TC's motion. The TC is weakened by its encounter with the water obstacle, and that may be a contributing factor to its slow postdeparture motion of $3.7 \mathrm{~m} \mathrm{~s}^{-1}$. In this case, the typhoon never regains the forward speed of its control ocean counterpart.

\section{Discussion and summary}

Data from 84 typhoons that made landfall on the eastern coast of Taiwan between 1960 and 2010 are analyzed. The storms were designated northern landfall typhoons (NLT) and southern landfall typhoons (SLT) based on their landfall position with respect to $23.5^{\circ} \mathrm{N}$. The typhoons are also classified as slow, medium, or fast movers with respect to the average overland translation speed for the 61 continuous-track storms. Among that subset, we find that $77 \%$ (60\%) of slow (fast) typhoons belongs to the NLT (SLT) group. On average, the slow (fast) cases often have a slower (faster) prelandfall speed and decelerate (accelerate) further during the landfall period. As a result, the average overland duration time of the slow cases is $16 \mathrm{~h}$, which is much longer than the 3-h mean duration of the fast cases. The combination of slower translation with longer duration for the northern class of TCs leads to large rainfall on the slope of the Central Mountain Range (CMR).

As in the observations, the NLT storms in our semiidealized WRF simulations tend to turn toward the island and accelerate in the hours preceding landfall, only to slow down substantially while over land. Previous studies (e.g., Jian and Wu 2008; Kuo et al. 2001) have advanced purely physical explanations for similar track deflections, involving blocking and channeling of the flow, the topographic $\beta$ effect, etc. Yet, the TC is a potential vorticity (PV) anomaly, and our PV-based analysis suggests that asymmetric diabatic heating associated with topography can also help explain the complex motions of these TCs as they approach and pass over the island. This is revealed by the PV equation's diabatic heating $(\mathrm{DH})$ term, which is based on gradients of diabatic heating $Q$ and absolute vorticity $\mathbf{q}$ and not on $Q$ itself (or even the surface rainfall field). ${ }^{5}$

Our approach is viable because convective activity represents the integrated result of many different factors and forcings, and the physical and thermodynamic aspects of a TC constitute a tightly coupled system. Introducing an obstacle like Taiwan in the path of a TC alters the flow, causing physical responses, such as ascent and descent, channeling, and blocking. But this also alters the convective heating by introducing variations in convergence and advection. The modulated convective heating itself then alters the flow, in important and powerful ways, which then further impacts the heating. Indeed, we see DH as the end product of all of these individual influences, physical and thermodynamic. As previous studies on the terrain effect did not consider whether convective heating variations could have played a role in the track deflections at all, our study provides a complementary mechanism for understanding shifts in typhoon motion near mountainous terrain, one that is definitely more general, and possibly even superior.

In contrast to its ocean control (OC) counterpart, the prelandfall NLT case T3N does develop azimuthal

\footnotetext{
${ }^{5}$ Keep in mind that DH and the other PV terms (such as HA, VA) are not independent and strongly modulate each other, as noted earlier.
} 
(a)

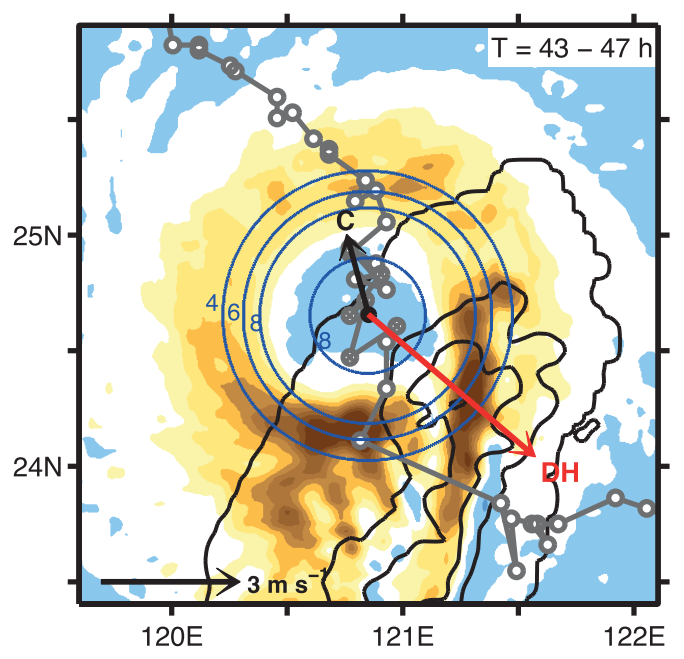

(c)

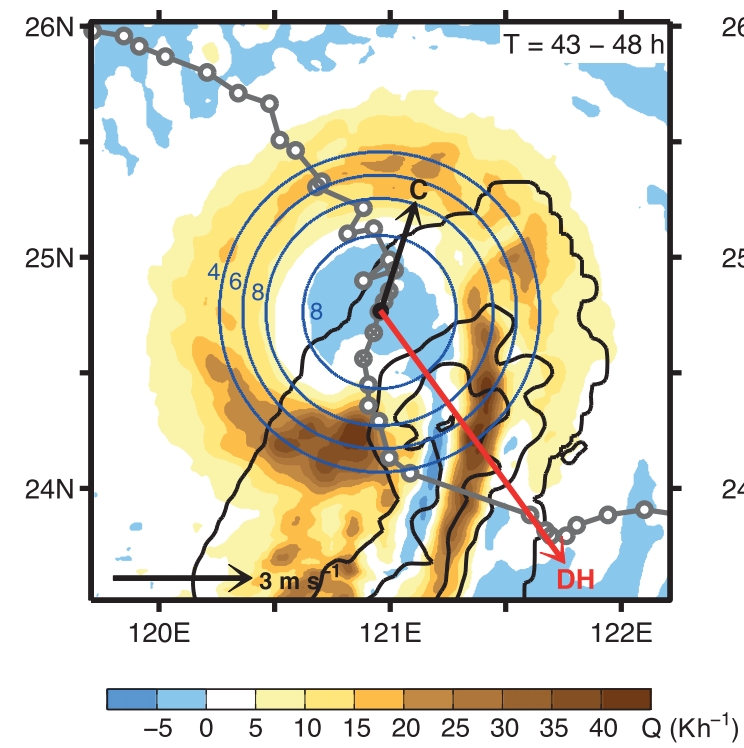

(b)

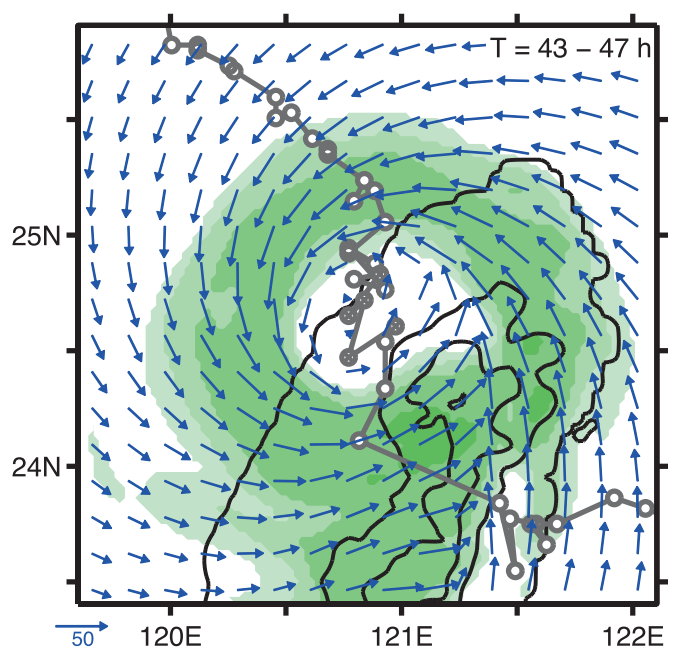

(d)

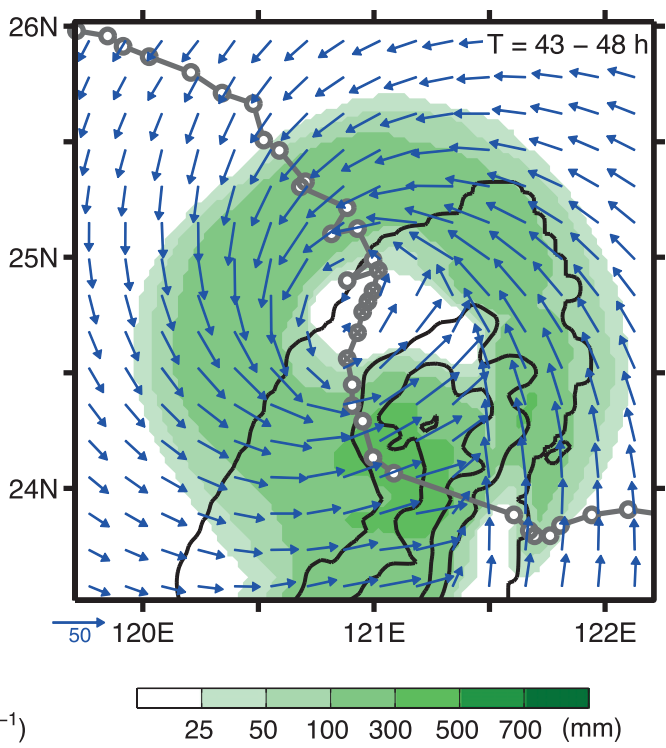

FIG. 11. As in Fig. 8, but for experiments employing initial vortices that are (a),(b) more intense $\left(V_{m}=65 \mathrm{~m} \mathrm{~s}^{-1}\right.$, experiment T3V65) and (c),(d) larger in size $\left(R_{m}=75 \mathrm{~km}\right.$, experiment T3R75).

latent heating variations as its circulation encounters the island that result in wavenumber-1 (WN1) asymmetries in the DH field. The DH term points toward landfall as cyclone $\mathrm{T} 3 \mathrm{~N}$ neared the coast, causing the storm to increase in speed and shift in direction. Once the NLT crosses the CMR, however, the topographically enhanced convection and diabatic heating shifts toward the typhoon's rear flank. During this period, DH is working to slow the storm, as it has a component opposing the northwestward motion encouraged by the combination of large-scale steering and beta drift. This process appears to explain why the model typhoons tend to decelerate overland, which further prolongs the TC's overland duration and increases the rainfall potential over the island.

In contrast, for the SLT, the DH contribution that develops while overland tends to point toward the storm's forward flank, hastening its transit across the island and resulting in relatively shorter overland durations and less total rainfall. This may explain why the SLT was dominated by fast movers that tended to increase translation speed during the overland period, 
(a)

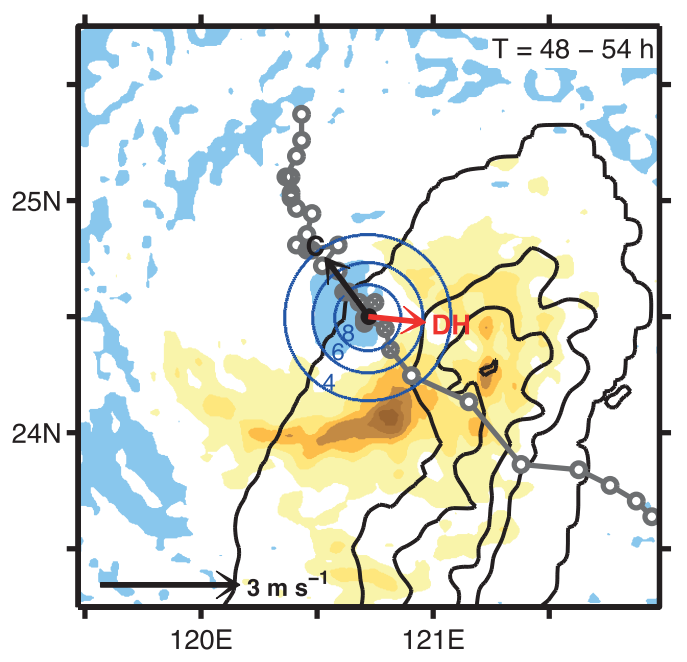

(b)

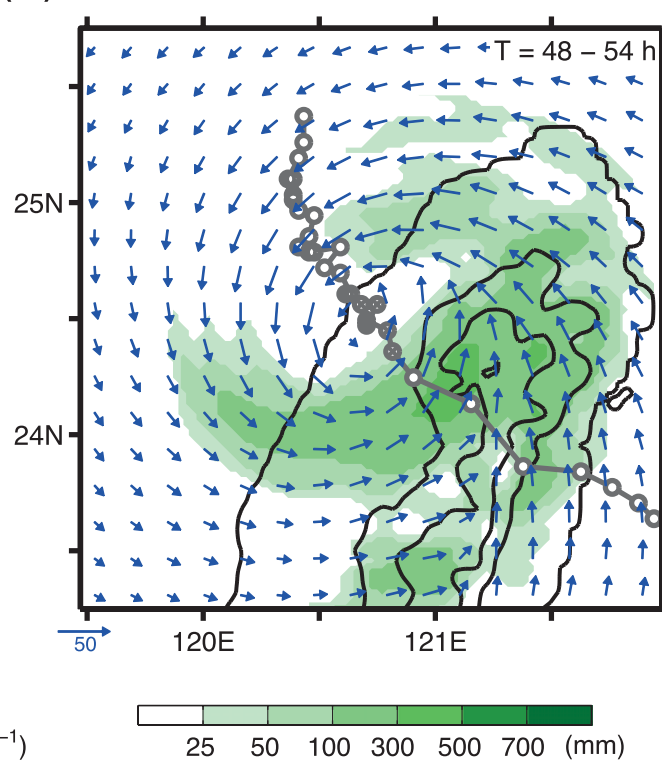

FIG. 12. As in Fig. 8, but for weaker initial storm $\left(V_{m}=25 \mathrm{~m} \mathrm{~s}^{-1}\right.$, experiment T3V25).

even for cases that maintained continuous tracks. We also saw that generally faster-moving NLT cases were slowed relatively less by the DH term, apparently because the storm motions in these cases were dominated more by the steering flow.

As a consequence, we see a positive feedback operating between the terrain-focused convection and the TC's motion, in that the translation speed of

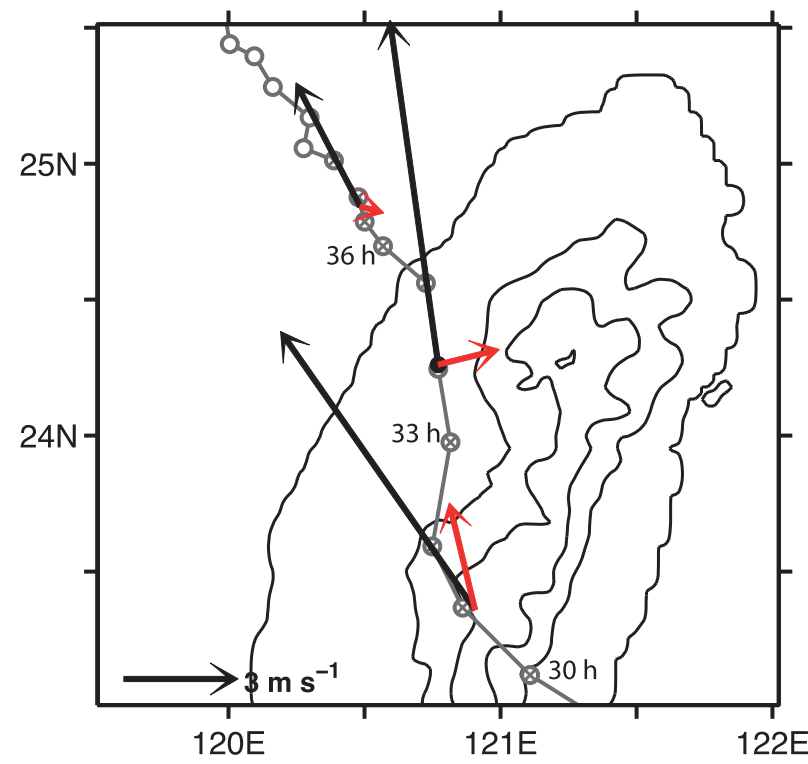

FIG. 13. Track of experiment T5S, showing storm motion (black) and DH term (red vectors) for various stages along the track Averaging intervals are 30-32, 33-35, and 36-39 h. slower-moving NLT storms can be further reduced, leading to additional asymmetric heating that supports even longer overland durations. After being drawn toward the island, these typhoons turn northward after passing over the CMR, a consequence of asymmetric heating associated with topography shifting around toward the storm's rear flank. The DH term's large magnitude is acting to reduce the translation speed, and its direction is fostering a track that keeps the TC moving parallel to-rather than away from-the mountains. Both serve to increase the amount of heating and precipitation that is generated over the CMR. As long as the storm continues to experience asymmetric heating forced by its own cyclonic circulation impinging on the topography, the translation away from the island remains opposed, and the slower its motion prior to landfall, the more susceptible to this effect the TC appears to be. The upshot is that the topographically phase-locked convection tends to causes the NLTs to linger much longer over land, permitting them to produce an impressively large rainfall amount.

Acknowledgments. This research was supported by the Central Weather Bureau, Taiwan, through Grants MOTC-CWB-96-2M-01, MOTC-CWB-97-2M-01, MOTCCWB-98-2M-01, MOTC-CWB-99-6M-02, and MOTCCWB-100-6M-02. We thank the Central Weather Bureau for its typhoon database (http://rdc28.cwb.gov.tw/) for providing the typhoon and rainfall data, and Dr. Shih-Hao $\mathrm{Su}$ for his helpful comments. 


\section{REFERENCES}

Bender, M. A., R. E. Tuleya, and Y. Kurihara, 1987: A numerical study of the effect of island terrain on tropical cyclones. Mon. Wea. Rev., 115, 130-155.

Brand, S., and J. Blelloch, 1974: Changes in the characteristics of typhoons crossing the island of Taiwan. Mon. Wea. Rev., 102, 708-713.

Cao, Y., R. G. Fovell, and K. L. Corbosiero, 2011: Tropical cyclone track and structure sensitivity to initialization in idealized simulations: A preliminary study. Terr. Atmos. Oceanic Sci., 22, 559-578.

Chan, J. C. L., F. M. F. Ko, and Y. M. Lei, 2002: Relationship between potential vorticity tendency and tropical cyclone motion. J. Atmos. Sci., 59, 1317-1336.

Chang, C.-P., T.-C. Yeh, and J. M. Chen, 1993: Effects of terrain on the surface structure of typhoons over Taiwan. Mon. Wea. Rev., 121, 734-752.

Chang, S. W. J., 1982: The orographic effects induced by an island mountain range on propagating tropical cyclones. Mon. Wea. Rev., 110, 1255-1270.

Cheung, K. K. W., L. R. Huang, and C. S. Lee, 2008: Characteristics of rainfall during tropical cyclone periods in Taiwan. Nat. Hazards Earth Syst., 8, 1463-1474.

Chien, F.-C., and H.-C. Kuo, 2011: On the extreme rainfall of Typhoon Morakot (2009). J. Geophys. Res., 116, D05104, doi:10.1029/2010JD015092.

Corbosiero, K. L., and J. Molinari, 2003: The relationship between storm motion, vertical wind shear, and convective asymmetries in tropical cyclones. J. Atmos. Sci., 60, 366-376.

Dudhia, J., 1989: Numerical study of convection observed during the winter monsoon experiment using a mesoscale twodimensional model. J. Atmos. Sci., 46, 3077-3107.

Fovell, R. G., and H. Su, 2007: Impact of cloud microphysics on hurricane track forecasts. Geophys. Res. Lett., 34, L24810, doi:10.1029/2007GL031723.

— K. L. Corbosiero, and H. C. Kuo, 2009: Cloud microphysics impact on hurricane track as revealed in idealized experiments. J. Atmos. Sci., 66, 1764-1778.

,-- A. Seifert, and K.-N. Liou, 2010: Impact of cloudradiative processes on hurricane track. Geophys. Res. Lett., 37, L07808, doi:10.1029/2010GL042691.

Holland, G. J., 1983: Tropical cyclone motion: Environmental interaction plus a beta effect. J. Atmos. Sci., 40, 328-342.

Ishijima, S., and M. Estoque, 1987: An observation study of orographic effects on westbound typhoon crossing Taiwan. J. Meteor. Soc. Japan, 65, 455-467.

Jian, G.-J., and C.-C. Wu, 2008: A numerical study of the track deflection of super-typhoon Haitang (2005) prior to its landfall in Taiwan. Mon. Wea. Rev., 136, 598-615.

Jordan, C. L., 1958: Mean soundings for the West Indies area. J. Meteor., 15, 91-97.
Kuo, H.-C., R. T. Williams, J.-H. Chen, and Y.-L. Chen, 2001: Topographic effects on barotropic vortex motion: No mean flow. J. Atmos. Sci., 58, 1310-1327.

Lee, C.-S., L.-R. Huang, H.-S. Shen, and S.-T. Wang, 2006: A climatology model for forecasting typhoon rainfall in Taiwan. Nat. Hazards, 37, 87-105.

Lin, Y.-L., R. D. Farley, and H. D. Orville, 1983: Bulk parameterization of the snow field in a cloud model. J. Climate Appl. Meteor., 22, 1065-1092.

—, S.-Y. Chen, C. M. Hill, and C.-Y. Huang, 2005: Control parameters for the influence of a mesoscale mountain range on cyclone track continuity and deflection. J. Atmos. Sci., 62, 1849-1866.

Mlawer, E. J., S. J. Taubman, P. D. Brown, M. J. Iacono, and S. A. Clough, 1997: Radiative transfer for inhomogeneous atmospheres: RRTM, a validated correlated- $k$ model for the longwave. J. Geophys. Res., 102 (D14), 16 663-16 682.

Shieh, S. L., S. T. Wang, M. D. Cheng, and T. C. Yeh, 1998: Tropical cyclone tracks over Taiwan and its vicinity for the one hundred years from 1897 to 1996 (in Chinese). Central Weather Bureau Research Rep. CWB86-1M- 01, 497 pp.

Su, S.-H., H.-C. Kuo, L.-H. Hsu, and Y.-T. Yang, 2012: Temporal and spatial characteristics of typhoon extreme rainfall in Taiwan. J. Meteor. Soc. Japan, 90, 721-736.

Tuleya, R. E., and Y. Kurihara, 1978: A numerical simulation of the landfall of tropical cyclones. J. Atmos. Sci., 35, 242-257.

— M. A. Bender, and Y. Kurihara, 1984: A simulation study of the landfall of tropical cyclones using a movable nested-mesh model. Mon. Wea. Rev., 112, 124-136.

Wang, S. T., 1980: Prediction of the behavior and intensity of typhoons in Taiwan and its vicinity (in Chinese). Chinese National Science Council Research Rep. 108, 100 pp.

Wong, M. L. M., and J. C. L. Chan, 2006: Tropical cyclone motion in response to land surface friction. J. Atmos. Sci., 63, 13241337.

— and _ 2007: Modeling the effects of land-sea roughness contrast on tropical cyclone winds. J. Atmos. Sci., 64, 32493264.

Wu, L., and B. Wang, 2000: A potential vorticity tendency diagnostic approach for tropical cyclone motion. Mon. Wea. Rev., 128, 1899-1911.

— and - 2001: Effects of convective heating on movement and vertical coupling of tropical cyclones: A numerical study. J. Atmos. Sci., 58, 3639-3649.

Yeh, T.-C., and R. L. Elsberry, 1993a: Interaction of typhoons with the Taiwan orography. Part I: Upstream track deflections. Mon. Wea. Rev., 121, 3193-3212.

— orography. Part II: Continuous and discontinuous tracks across the island. Mon. Wea. Rev., 121, 3213-3233.

Zehnder, J. A., 1993: The influence of large-scale topography on barotropic vortex motion. J. Atmos. Sci., 50, 2519-2532. 